\title{
Books received*
}

Haemoglobin: Structure, Function, Evolution, and Pathology, by Richard E. Dickerson and Irving Geis, published by the Benjamin/Cummings Publishing Company, Inc., Menlo Park, California, price £22.45. ISBN 0805324119.

Genetic Recombination, by Harold L. K. Whitehouse, published by John Wiley \& Sons Limited, Baffins Lane, Chichester, West Sussex, England P019 1UD, price £23.75. ISBN 0471102059 .

Cyclodextrins and their Inclusion Complexes, by J. Szejtli, published by Akademiai

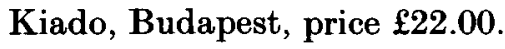

Delayed and Chronic Effects of Chemicals in the Workplace (Report on a WHO Meeting). Copenhagen, WHO Regional Office for Europe, price Sw. Fr. 4. ISBN 9289012307.

Current Awareness in Biological Sciences, vol. 100, no. 1, March 1983. A new journal published monthly by Pergamon Press Limited, Headington Hill Hall, Oxford OX3 0BW, England. Annual subscription $1983 \$ 1,500,2$ years 1983/1984 $\$ 2,850$.

Nitrosamines and Human Cancer, Banbury Report 12, edited by Peter N. Magee, Temple University School of Medicine, published by Cold Spring Harbor Laboratory, Fulfillment Department, P.O. Box 100, Cold Spring Harbor, NY 11724 , price $\$ 67$ ( $\$ 80.40$ outside U.S.). ISSN 0198006812 .

Biotechnology, A Comprehensive Treatise in 8 Volumes, edited by H.-J. Rehm and G. Reed, volume 3 : Biomass, Microorganismsfor Special Applications, Microbial Products I, Energy from Renewable Resources, published by Verlag Chemie, Weinheim, Deerfield Beach, Florida, Basel, price DM 495.

Structures of DNA, Cold Spring Harbor Symposia on Quantitative Biology, volume XLVII, published by Cold Spring Harbor Laboratory, Fulfillment Department, P.O. Box 100, Cold Spring Harbor, NY 11724, price $\$ 140$ ( $\$ 168$ outside US). ISBN 0879690461.

The Arctic Skua by Peter O'Donald, published by Cambridge University Press, The Edinburgh Building, Shaftesbury Road, Cambridge CB2 2RU, price £25.00. ISBN 0521235812.

Indicators of Genotoxic Exposure, Banbury Report 13, edited by Bryn A. Bridges, Byron E. Butterworth and I. Bernard Weinstein, published by Cold Spring Harbor Laboratory, Fulfillment Department, P.O. Box 100, Cold Spring Harbor, NY 11724, price \$62.50 US, \$75 elsewhere. ISBN $087969212 \mathrm{X}$.

Drug Resistance in Bacteria, edited by Susumu Mitsuhashi, published by Japan Scientific Societies Press, Tokyo/Thieme-Stratton Inc. New York, price DM 148. ISBN 3136414012 (TSI).

* Books in this list may be revised in later numbers of the journal. 